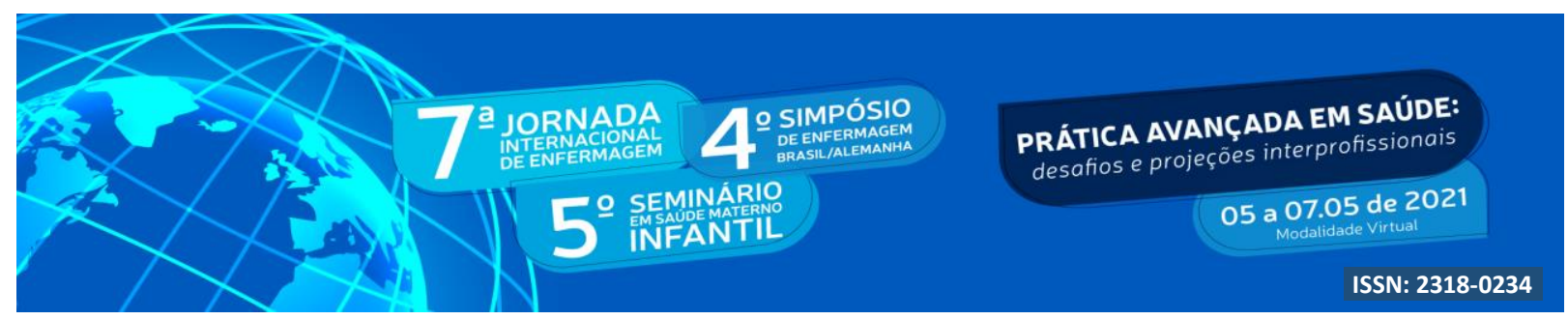

DOI: http://doi.org/10.48195/jie2021-136

\title{
ANÁLISE DO NÍVEL DE COMPAIXÃo DOS PROFISSIONAIS DE ENFERMAGEM EM TEMPOS DE PANDEMIA ${ }^{1}$
}

\section{Layana Santos Silva ${ }^{2}$; Anderson Varjão de Oliveira ${ }^{3}$; Renata Santos Solheiro ${ }^{4}$; Yone Gonçalves dos Santos ${ }^{5}$; Sheron Brandão da Silva ${ }^{6}$; Vinicius Lino de Souza Neto ${ }^{7}$.}

\begin{abstract}
RESUMO
Nesse sentido, este estudo tem como objetivo analisar a compaixão dos profissionais de enfermagem em tempos de pandemia. Realizou-se uma pesquisa de revisão integrativa da literatura, utilizando publicações científicas disponibilizadas nas bases de dados LILACS, Medline e BDENF, que se buscou por meio dos Descritores em Ciências da Saúde (DeCS) que foram analisados de forma pareada, alguns aspectos como metodologia e nível de evidência cientifica, com amostra final de 15 artigos. Os resultados mostram que os fatores relacionados pontuam que a compaixão gera fadiga decorrente de tais situações, como: as condições de trabalho, as emoções, os fatores psicológicos e suas características sociodemográficas. A presença de exposição a fadiga da compaixão pode levar a várias doenças físicas e mentais, mas também ocasiona um declínio no compromisso com o trabalho, resultando em uma queda no entusiasmo, baixa eficiência e até mesmo negligência.
\end{abstract}

Palavras-chave: Condições de Trabalho; Enfermagem; Sofrimento por Compaixão; Relações Interpessoais; Saúde Mental.

\begin{abstract}
In this sense, this study aims to analyze the compassion of nursing professionals in times of pandemic. An integrative literature review research was carried out, using scientific publications made available in the LILACS, Medline and BDENF databases, which were searched through the Health Sciences Descriptors (DeCS) which were analyzed in a paired way, some aspects as a methodology and level of scientific evidence, with a final sample of 15 articles. The results show that the related factors point out that compassion generates fatigue due to such situations, such as: working conditions, emotions, psychological factors and their sociodemographic characteristics. The presence of exposure to compassion fatigue can lead to various physical and mental illnesses, but it also causes a decline in commitment to work, resulting in a drop in enthusiasm, low efficiency and even negligence.
\end{abstract}

Key Words: Interpersonal Relations; Mental health; Nursing; Suffering for Compassion; Work conditions.

\footnotetext{
${ }^{1}$ Revisão integrativa.

${ }^{2}$ Estudante do Curso de Enfermagem. Universidade nove de julho. E-mail: layanasasilva@ gmail.com

${ }^{3}$ Estudante do Curso de Enfermagem. Universidade nove de julho. E-mail: andersondiscord @ outlook.com

${ }^{4}$ Estudante do Curso de Enfermagem. Universidade nove de julho. E-mail: $\underline{\text { solheirorenata@ gmail.com }}$

${ }^{5}$ Estudante do Curso de Enfermagem. Universidade nove de julho. E-mail: yonegoncalves16@gmail.com

${ }^{6}$ Estudante do Curso de Enfermagem. Universidade nove de julho. E-mail: sheron.brandao.sb@gmail.com

${ }^{7}$ Orientador. Enfermeiro, Doutorando. Universidade noye de julho. E-mail: vinolino@ hotmail.com
} 


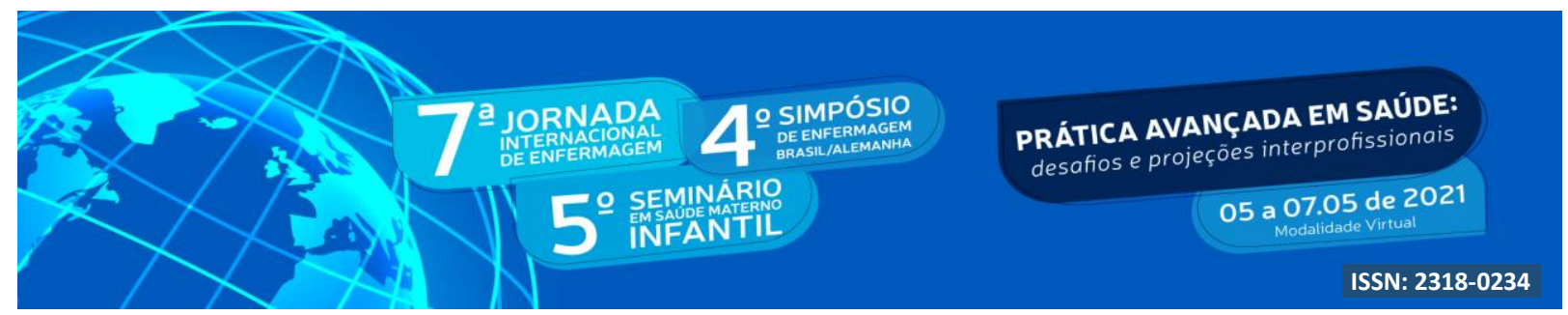

\section{INTRODUÇÃO}

As pessoas são, em muitos aspectos, indivíduos que buscam seus objetivos pessoais e profissionais, além disso, são membros de coletivos sociais que se unem uns aos outros. E segundo Forsyth \& Burnette (2010), a tendência de se conectar com outras pessoas é talvez a característica individual mais importante da humanidade. Entretanto, a percepção da assistência qualificada para os pacientes acometidos pelo COVID-19 é influenciada pela qualidade das relações interpessoais, pela compaixão, profissionais abertos à fala e a escuta e validação das informações que são compartilhadas (OLIVEIRA E PASSOS, 2020).

Marçal (1994) menciona que, cuidar é o jeito humano de ser, o que significa que o profissional de enfermagem cuida do outro não somente por ele ser um profissional, mas também porque é uma pessoa. Rincón Álvarez (2013), caracteriza sete particularidades implícitas no ato de cuidar em enfermagem, são elas: compaixão, competência, confiança, consciência, compromisso, comportamento e criatividade.

A origem da palavra latina para compaixão é passio, a qual, significando "sofrer" pode acrescentar o prefixo com "sofre com" para uma melhor compreensão (LILIUS et al., 2008). Nessa perspectiva, o significado de compaixão é compartilhado com outra pessoa, ou seja, se colocar no lugar do outro, sentir sua dor como sendo nossa e demonstrar gentilmente o seu ponto de vista. Karen Armstrong (2011) apresenta a compaixão fundamentada em conceitos satélites, como a empatia e o sofrimento, em que o enfermeiro se vê em grande parte em sua prática profissional.

Ademais, a fadiga da compaixão, por outro lado, parece ser específica à profissionais de saúde, constantemente expostos ao sofrimento humano e a intensas jornadas acerca do cuidado (ZENKNER et al., 2020). Batalha e colaboradores (2019) desenvolveram um estudo quantitativo do tipo descritivo e transversal, nele é evidenciado os níveis médio e alto da Fadiga por Compaixão nos profissionais de saúde.

Nesse contexto, acredita-se que a fadiga decorrente da compaixão é um problema vivenciado pelos profissionais de enfermagem e que alguns fatores contribuem para isso. Os 


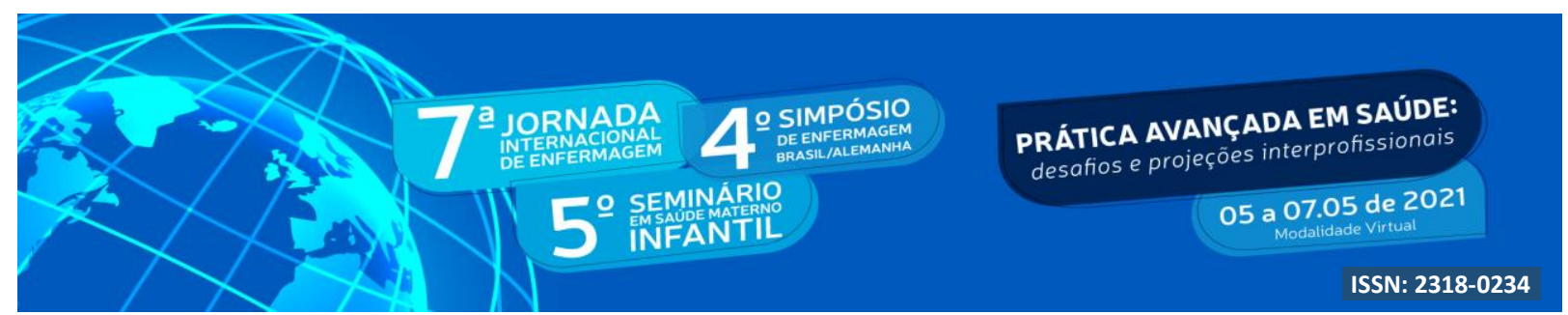

profissionais de saúde que estão principalmente na linha de frente no combate a Covid-19 experimentam altos níveis de esgotamento e fadiga da compaixão, afetando sua saúde e os cuidados que prestam.

\section{OBJETIVO}

Este estudo teve como objetivo analisar a compaixão dos profissionais de enfermagem em tempos de pandemia.

\section{METODOLOGIA}

Realizou-se uma revisão integrativa da literatura e foram obedecidas as seis etapas do processo de elaboração da revisão integrativa, expostas a seguir: elaboração da pergunta norteadora, estabelecimento dos objetivos da revisão e critérios de inclusão e exclusão dos artigos; definição das informações a serem extraídas das pesquisas; seleção dos artigos na literatura; análise dos resultados; discussão dos achados e apresentação da revisão (WHITTEMORE e KNAFL, 2005).

Para conduzir esta revisão, formulou-se a seguinte questão norteadora: Qual a relação entre a compaixão vivida pelos profissionais de enfermagem no cenário de pandemia? Para a seleção dos artigos utilizou-se o acesso on-line às bases de dados da Biblioteca Virtual da Saúde (BVS), Sistema Latino-Americano e do Caribe de informação em Ciências da Saúde (LILACS), Banco de dados de Enfermagem (BDENF) e Scientific Electronic Library Online (SciELO).

Para o levantamento das publicações foram utilizados os descritores cadastrados nos Descritores em Ciências da Saúde (DeCS) e do Medical Subject Headings (MeSH): “compaixão", "Covid-19", “fadiga por compaixão", "satisfação por compaixão" e "Saúde Mental" (em português), sendo combinados por meio do conector booliano "AND" com o descritor "Enfermagem", inseridos através do formulário para busca avançada.

A pesquisa foi realizada nos meses de janeiro a março de 2021. Os critérios de inclusão utilizados para a seleção dos artigos analisados foram: artigos completos e 


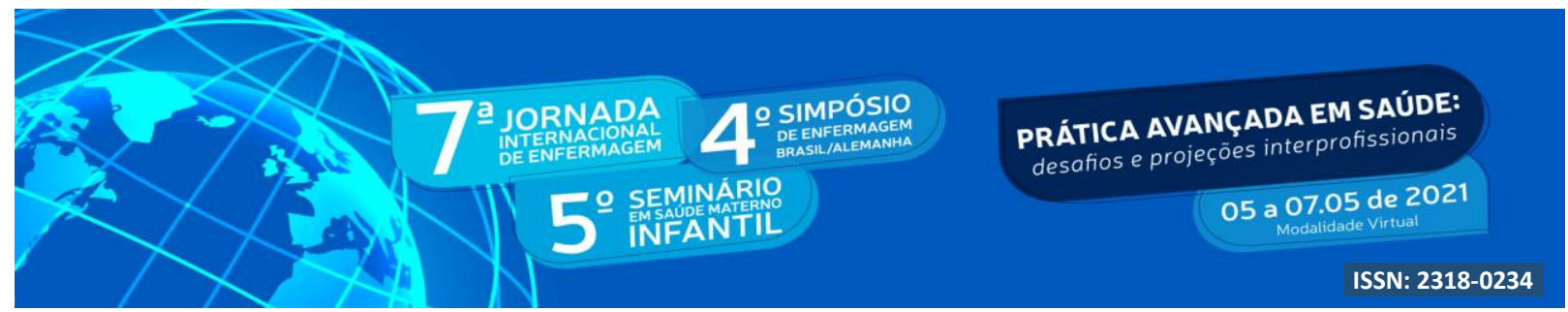

disponíveis que discutiam a importância da compaixão realizada pelos profissionais de enfermagem na saúde mental e frente ao COVID-19. Os critérios de exclusão aplicados foram: artigos em formato de editoriais. Destaca-se que o recorte temporal do último ano é uma estratégia recomendada para a qualificação da pesquisa.

Para realizar a análise da amostra, utilizou-se um instrumento adaptado (Quadro 1), que contemplou os seguintes aspectos: Título do artigo, periódico, ano, delineamento e objetivo. Os autores utilizados neste estudo foram devidamente referenciados, respeitando e identificando as fontes de pesquisa, observando rigor ético quanto à propriedade intelectual dos textos científicos que foram analisados, no que se refere ao uso do conteúdo e de citação das partes das obras consultadas.

No intuito de manter nesta investigação estudos de qualidade, os artigos préselecionados (Quadro 1) foram avaliados como relevantes e metodologicamente adequados, utilizando um formulário para avaliação de estudos elaborados pelo Critical Appraisal Skills Programme (CASP) (BHATTACHERJEE, 2002). Os estudos que atingiram um escore de 7 , do máximo possível de 10 pontos, foram incluídos na amostra.

Após o procedimento da busca eletrônica nas bases de dados mencionadas, realizou-se uma avaliação dos artigos por quatro revisores, sendo posteriormente comparados os resultados, a fim de certificar que os artigos atendiam aos critérios de inclusão. Dessa forma, as publicações foram pré-selecionadas com base na leitura do título e resumo. Em seguida, procedeu-se à leitura na íntegra dos artigos previamente selecionados, identificando os artigos que compuseram a amostra final desta revisão integrativa.

A amostra inicial foi de 50 artigos, no entanto, após a aplicação dos critérios estabelecidos, obteve-se uma amostra final de 15 estudos.

\section{RESULTADOS E DISCUSSÃO}

O Quadro 1 sintetiza as informações disponibilizadas pelos artigos que foram incluídos na revisão da literatura.

Quadro 1 - Relação dos artigos que pertencem ao eixo da temática em questão, conforme critérios, São Paulo/SP, 2021. 


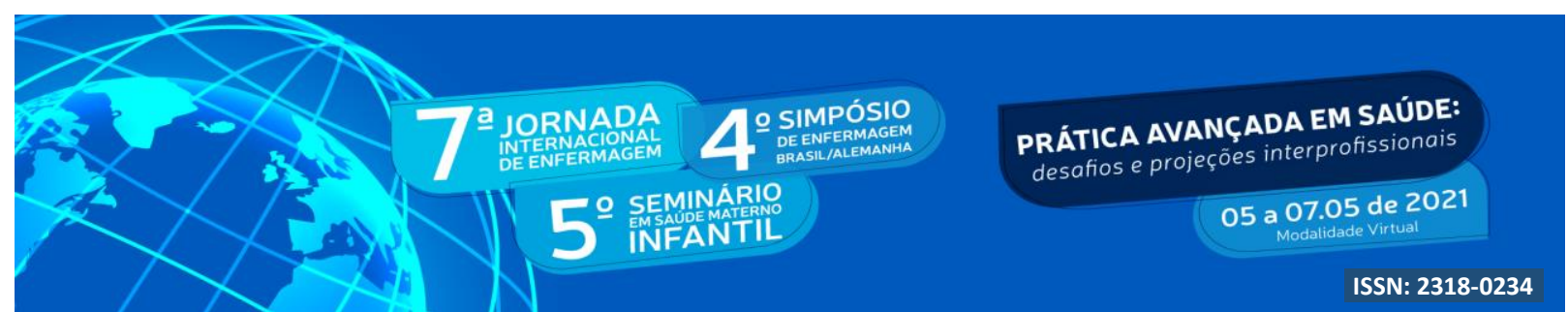

\begin{tabular}{|c|c|c|c|c|}
\hline Título do Artigo & Periódico & Ano & Delineamento & Objetivo \\
\hline $\begin{array}{l}\text { Relationship } \\
\text { Between } \\
\text { Compassion } \\
\text { Fatigue in } \\
\text { Nurses, and } \\
\text { Work-Related } \\
\text { Stress and the } \\
\text { Meaning of Life }\end{array}$ & $\begin{array}{l}\text { Journal of } \\
\text { Religion and } \\
\text { Health }\end{array}$ & 2021 & $\begin{array}{l}\text { Estudo } \\
\text { transversal }\end{array}$ & $\begin{array}{l}\text { Examinar a correlação entre o } \\
\text { estresse relacionado ao trabalho e o } \\
\text { sentido da vida em associação com } \\
\text { a fadiga da compaixão de } \\
\text { enfermeiros, bem como para } \\
\text { determinar os fatores que afetam a } \\
\text { fadiga da compaixão. }\end{array}$ \\
\hline $\begin{array}{l}\text { Prevalence and } \\
\text { predictors for } \\
\text { compassion } \\
\text { fatigue and } \\
\text { compassion } \\
\text { satisfaction in } \\
\text { nursing students } \\
\text { during clinical } \\
\text { placement }\end{array}$ & $\begin{array}{l}\text { Nurse } \\
\text { Education in } \\
\text { Practice }\end{array}$ & 2021 & $\begin{array}{l}\text { Estudo } \\
\text { transversal }\end{array}$ & $\begin{array}{l}\text { Explorar as relações entre apoio } \\
\text { social, empatia, resiliência, } \\
\text { estratégias de enfrentamento, } \\
\text { fadiga e satisfação por compaixão } \\
\text { em estudantes de enfermagem } \\
\text { durante a colocação clínica na } \\
\text { China. }\end{array}$ \\
\hline $\begin{array}{l}\text { Prevalence and } \\
\text { factors of } \\
\text { compassion } \\
\text { fatigue among } \\
\text { nurse in China }\end{array}$ & Medicine & 2121 & $\begin{array}{l}\text { Revisão } \\
\text { sistemática }\end{array}$ & $\begin{array}{l}\text { Fornecer uma avaliação abrangente } \\
\text { das evidências empíricas atuais } \\
\text { para a prevalência e os fatores de } \\
\text { fadiga da compaixão entre } \\
\text { enfermeiras clínicas chinesas. }\end{array}$ \\
\hline $\begin{array}{l}\text { The impact of } \\
\text { resilience on } \\
\text { turnover } \\
\text { intention in } \\
\text { dialysis nurses: } \\
\text { The mediating } \\
\text { effects of work } \\
\text { engagement and } \\
\text { compassion } \\
\text { fatigue }\end{array}$ & $\begin{array}{l}\text { Japan } \\
\text { Journal of } \\
\text { nursing } \\
\text { science }\end{array}$ & 2021 & $\begin{array}{l}\text { Estudo } \\
\text { transversal } \\
\text { descritivo }\end{array}$ & $\begin{array}{l}\text { Descrever os níveis de intenção de } \\
\text { rotatividade e explorar os efeitos } \\
\text { mediadores do envolvimento no } \\
\text { trabalho e da fadiga por compaixão } \\
\text { na relação entre resiliência e } \\
\text { intenção de rotatividade em } \\
\text { enfermeiras de diálise. }\end{array}$ \\
\hline $\begin{array}{l}\text { The Relationship } \\
\text { Between Present- } \\
\text { Centered } \\
\text { Awareness and } \\
\text { Attention, } \\
\text { Burnout, and } \\
\text { Compassion } \\
\text { Fatigue in } \\
\text { Oncology Health } \\
\text { Professionals }\end{array}$ & Mindfulness & 2021 & $\begin{array}{l}\text { Estudo } \\
\text { qualitativo }\end{array}$ & $\begin{array}{l}\text { Determinar o papel de um tipo } \\
\text { específico de atenção plena, } \\
\text { consciência e atenção centradas no } \\
\text { presente, examinando sua relação } \\
\text { com o esgotamento e a fadiga da } \\
\text { compaixão entre os profissionais de } \\
\text { saúde oncológicos. }\end{array}$ \\
\hline
\end{tabular}




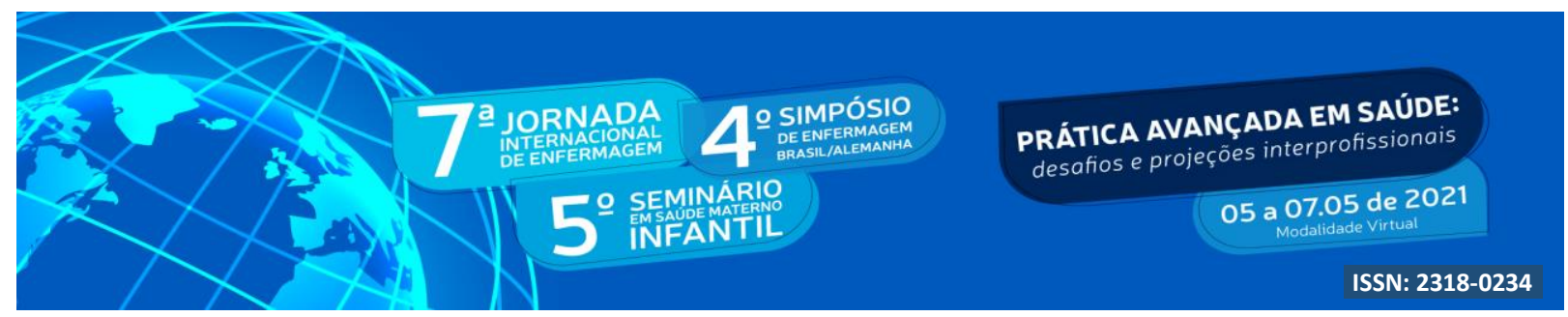

\begin{tabular}{|c|c|c|c|c|}
\hline $\begin{array}{l}\text { Predictors of } \\
\text { compassion } \\
\text { fatigue, burnout, } \\
\text { and compassion } \\
\text { satisfaction } \\
\text { among } \\
\text { emergency } \\
\text { nurses: A cross- } \\
\text { sectional survey }\end{array}$ & $\begin{array}{l}\text { International } \\
\text { Emergency } \\
\text { Nursing }\end{array}$ & 2021 & $\begin{array}{l}\text { Estudo de corte } \\
\text { transversal }\end{array}$ & $\begin{array}{l}\text { Desenvolver e testar um modelo } \\
\text { que estabeleça a relação preventiva } \\
\text { que a satisfação no trabalho, os } \\
\text { fatores individuais e relacionados } \\
\text { ao ambiente compartilham com a } \\
\text { fadiga da compaixão, o } \\
\text { esgotamento e a satisfação pela } \\
\text { compaixão. }\end{array}$ \\
\hline $\begin{array}{l}\text { Traumatic } \\
\text { Events Among } \\
\text { Cancer Patients } \\
\text { That Lead to } \\
\text { Compassion } \\
\text { Fatigue in } \\
\text { Nurses: A } \\
\text { Qualitative Study }\end{array}$ & $\begin{array}{l}\text { Journal of } \\
\text { Pain and } \\
\text { Symptom } \\
\text { Management }\end{array}$ & 2020 & $\begin{array}{l}\text { Estudo } \\
\text { qualitativo }\end{array}$ & $\begin{array}{l}\text { Descrever os componentes e as } \\
\text { frequências de eventos traumáticos } \\
\text { vivenciados por pacientes com } \\
\text { câncer, que, consequentemente, dão } \\
\text { origem ao cansaço da compaixão } \\
\text { do enfermeiro. }\end{array}$ \\
\hline $\begin{array}{l}\text { Enfermería, } \\
\text { pandemia } \\
\text { fatiga y } \\
\text { compasión: una } \\
\text { reflex-ión } \\
\text { general sobre el } \\
2020\end{array}$ & $\begin{array}{l}\text { Revista } \\
\text { Ciencia y } \\
\text { Cuidado }\end{array}$ & 2020 & $\begin{array}{l}\text { Estudo teórico } \\
\text { reflexivo }\end{array}$ & $\begin{array}{l}\text { Analisar os efeitos das atuais } \\
\text { condições de trabalho dos } \\
\text { profissionais de enfermagem e o } \\
\text { cansaço da compaixão, que } \\
\text { constitui um comprometimento da } \\
\text { qualidade de vida. }\end{array}$ \\
\hline $\begin{array}{l}\text { Mental health of } \\
\text { health } \\
\text { professionals: the } \\
\text { illness of those } \\
\text { who are } \\
\text { dedicated to } \\
\text { caring for the } \\
\text { illness of others }\end{array}$ & $\begin{array}{l}\text { Research, } \\
\text { Society and } \\
\text { Developmen } \\
\text { t }\end{array}$ & 2020 & $\begin{array}{l}\text { Revisão } \\
\text { bibliográfica } \\
\text { não sistemática } \\
\text { da literatura }\end{array}$ & $\begin{array}{l}\text { Discutir sobre o adoecimento dos } \\
\text { profissionais de saúde, abordando o } \\
\text { aumento significativo do } \\
\text { desenvolvimento de transtornos } \\
\text { mentais comuns } \\
\text { profissionais. }\end{array}$ \\
\hline $\begin{array}{lr}\text { Satisfação } & \mathrm{e} \\
\text { fadiga } & \text { por } \\
\text { compaixão em } \\
\text { Enfermeiros da } \\
\text { área hospitalar: } \\
\text { resultados } \\
\text { preliminares. } \\
\end{array}$ & $\begin{array}{l}\text { International } \\
\text { Congress of } \\
\text { Occupationa } \\
1 \quad \text { Health } \\
\text { Nursing- } \\
\text { ICOHN19: } \\
\text { Proceedings. }\end{array}$ & 2019 & $\begin{array}{l}\text { Estudo } \\
\text { quantitativo, do } \\
\text { tipo descritivo e } \\
\text { transversal. }\end{array}$ & $\begin{array}{l}\text { Identificar o nível de Satisfação e } \\
\text { Fadiga por Compaixão em } \\
\text { enfermeiros que atuam no contexto } \\
\text { hospitalar. }\end{array}$ \\
\hline $\begin{array}{lr}\text { Fadiga } & \text { por } \\
\text { compaixão } & \text { em } \\
\text { enfermeiros } & \text { de } \\
\text { urgência } & \text { e } \\
\text { emergência } & \\
\text { hospitalar } & \text { de } \\
\text { adultos } & \\
\end{array}$ & $\begin{array}{l}\text { Rev. Latino- } \\
\text { Americana } \\
\text { de } \\
\text { Enfermagem }\end{array}$ & 2019 & $\begin{array}{l}\text { Trata-se de um } \\
\text { estudo } \\
\text { quantitativo, } \\
\text { descritivo } \\
\text { transversal. }\end{array}$ & $\begin{array}{l}\text { Avaliar o nível de fadiga por } \\
\text { compaixão em enfermeiros e sua } \\
\text { associação em função de } \\
\text { características sociodemográficas e } \\
\text { profissionais. }\end{array}$ \\
\hline $\begin{array}{l}\text { Emotional } \\
\text { impact and } \\
\text { compassion }\end{array}$ & $\begin{array}{l}\text { European } \\
\text { Journal of } \\
\text { Oncology }\end{array}$ & 2019 & $\begin{array}{l}\text { Estudo } \\
\text { transversal. }\end{array}$ & $\begin{array}{l}\text { Avaliar a prevalência de } \mathrm{SC}, \mathrm{FC} \text { e } \\
\text { ansiedade em enfermeiras } \\
\text { oncológicas. }\end{array}$ \\
\hline
\end{tabular}




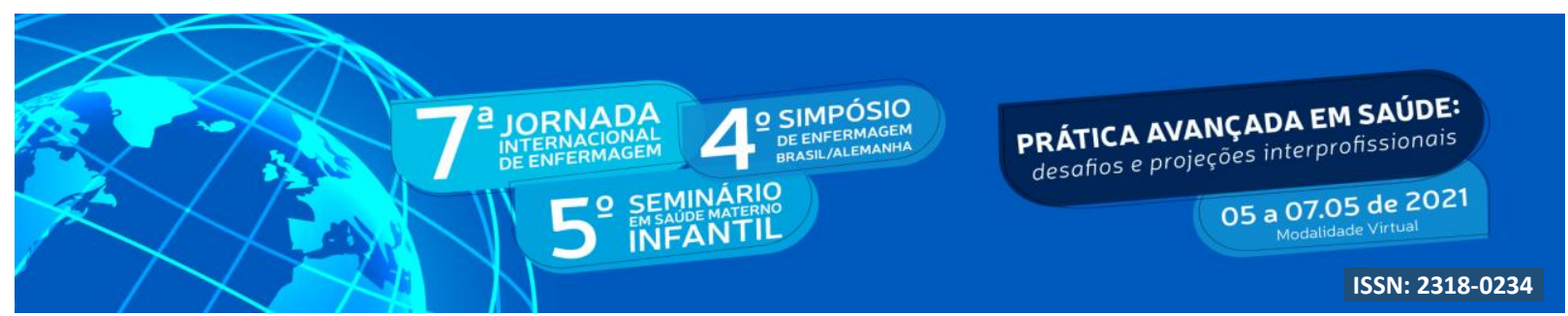

\begin{tabular}{|c|c|c|c|c|}
\hline $\begin{array}{l}\text { fatigue in } \\
\text { oncology nurses: } \\
\text { Results of a } \\
\text { multicentre study }\end{array}$ & Nursing & & & \\
\hline $\begin{array}{lr}\text { Fatigue } & \text { and } \\
\text { Stress as } & \text { a } \\
\text { predictor } & \text { of } \\
\text { Burnout } & \text { in } \\
\text { Health Care } & \\
\text { Professionals }\end{array}$ & $\begin{array}{l}\text { Revista } \\
\text { Psicologia } \\
\text { Organizaçõe } \\
\text { s e Trabalho }\end{array}$ & 2019 & $\begin{array}{l}\text { Estudo } \\
\text { correlacional e } \\
\text { de comparação } \\
\text { entre } \\
\text { participantes. }\end{array}$ & $\begin{array}{l}\text { Entender em que medida os efeitos } \\
\text { da fadiga e do estresse } \\
\text { desencadeiam a Síndrome de } \\
\text { Burnout em profissionais da saúde. }\end{array}$ \\
\hline $\begin{array}{lr}\text { Compassion } & \\
\text { Fatigue } & \text { in } \\
\text { Palliative } & \text { Care } \\
\text { Nursing: } & \text { A } \\
\text { Concept } & \\
\text { Analysis } & \\
\end{array}$ & $\begin{array}{l}\text { Journal of } \\
\text { hospice and } \\
\text { palliative } \\
\text { nursing }\end{array}$ & 2019 & $\begin{array}{l}\text { Revisão } \\
\text { integrativa da } \\
\text { literatura. }\end{array}$ & $\begin{array}{l}\text { Definir a fadiga da compaixão no } \\
\text { contexto da enfermagem em } \\
\text { cuidados paliativos. }\end{array}$ \\
\hline $\begin{array}{l}\text { A survey of } \\
\text { compassion } \\
\text { satisfaction, } \\
\text { burnout and } \\
\text { compassion } \\
\text { fatigue in nurses } \\
\text { practicing in } \\
\text { three oncology } \\
\text { departments in } \\
\text { Durban, South } \\
\text { Africa. }\end{array}$ & $\begin{array}{l}\text { International } \\
\text { Journal of } \\
\text { Africa } \\
\text { Nursing } \\
\text { Sciences }\end{array}$ & 2018 & $\begin{array}{l}\text { Uma pesquisa } \\
\text { quantitativa, não } \\
\text { experimental e } \\
\text { descritiva. }\end{array}$ & $\begin{array}{l}\text { Desenvolver uma intervenção nas } \\
\text { instalações para gerenciar a fadiga } \\
\text { da compaixão em enfermeiras } \\
\text { oncológicas. }\end{array}$ \\
\hline
\end{tabular}

Fonte: Fonte: Sistema Latino Americano e do Caribe de informação em Ciências da Saúde (LILACS), Scientific Electronic Library Online (SciELO) e Banco de dados de Enfermagem (BDENF), Medical Literature Analysisand Retrievel System Online (MEDLINE).

O nosso estudo identificou que o profissional com mais predisposição para desenvolver fadiga por compaixão são os enfermeiros. Visto que, vários estudos mostraram que o custo emocional de cuidar de pessoas em sofrimento destaca a ligação entre a fadiga por compaixão e o estresse no trabalho, especialmente quando é crônico e se transforma na síndrome de Burnout (ASLAN et al., 2021).

Além disso, os jovens profissionais apresentaram menor satisfação com a compaixão e maior estresse traumático secundário, com resultados semelhantes aos de outros pesquisadores (BORGES et al., 2019), isso pode ter sido ocasionado devido à sua inexperiência e fácil identificação com os pacientes. Portanto, esse fator indica que a fadiga da compaixão diminuirá com os anos de experiência profissional, de tal forma que pode estar 


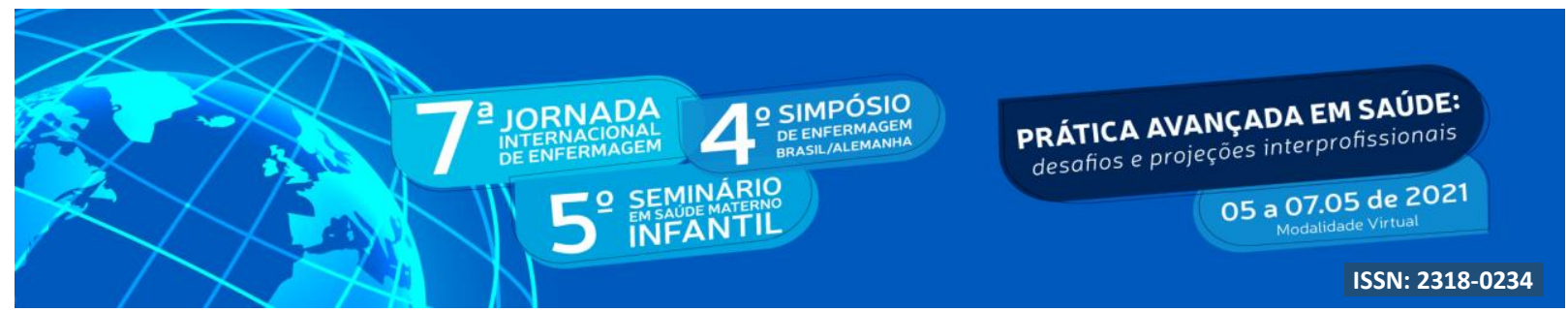

relacionado a uma adaptabilidade ainda não tão aparente em enfermeiros menos experientes.

Estudos anteriores apresentaram descobertas na relação entre o ambiente de trabalho e sua incidência no aparecimento de condições como a fadiga da compaixão, apesar de não serem pesquisas totalmente claras (CÁCERES-RIVERA, 2021). Alguns estudos sugerem que as condições de trabalho podem dar origem a sentimentos positivos e negativos, ou seja, o desenvolvimento de habilidades de liderança adequadas e o reconhecimento e gratidão no local de trabalho podem ser fatores de proteção. Além disso, um programa de incentivo profissional, acadêmico e até financeiro, seria uma ferramenta adequada para proteger os profissionais de enfermagem dos efeitos do cansaço relacionados à situação atual da pandemia da Covid-19.

Portanto, enfermeiros e administradores hospitalares podem ser lembrados de que é essencial monitorar a saúde mental dos profissionais de saúde para que seu estado emocional e psicológico não seja muito afetado pelo cuidado que prestam aos pacientes e possam, assim, manter um nível ideal para desempenhar o seu trabalho.

\section{CONCLUSÃO}

Observa-se que por meio da análise dos estudos o acometimento de profissionais de saúde que atuam no enfrentamento do Covid-19 pela síndrome da fadiga por compaixão está relacionado a fatores laborais e individuais. A presença de exposição a fadiga da compaixão pode levar a várias doenças físicas e mentais, mas também ocasiona um declínio no compromisso com o trabalho, resultando assim, em quedas no entusiasmo, baixa eficiência e até mesmo negligência, afetando negativamente o atendimento ao paciente e os resultados organizacionais.

Nesse sentido, é necessário elaborar uma linha de cuidado ou melhor aparelhar os serviços de saúde que implementam uma atenção psicológica, social e interativa, para que assim possa minimizar os possíveis efeitos da pandemia.

\section{REFERÊNCIAS}




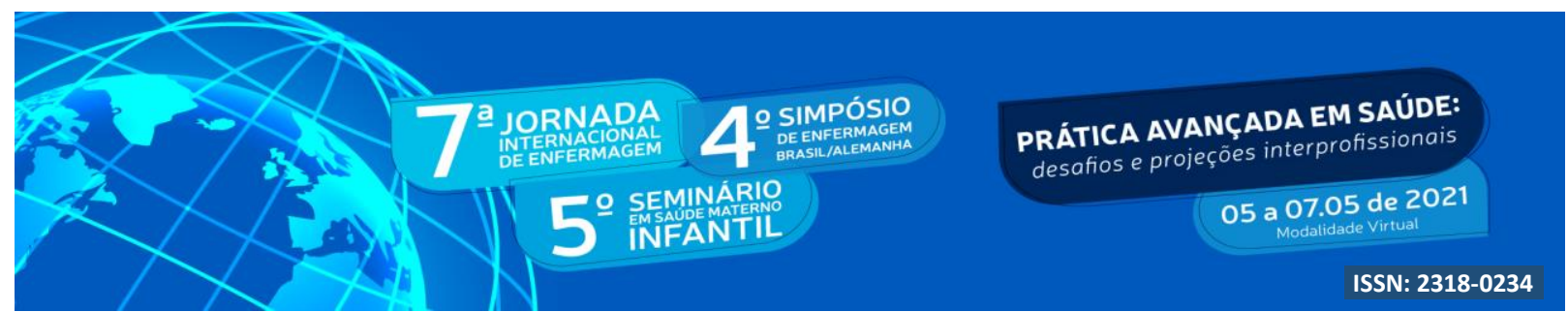

ARIMON-PAGÈS, E. et al. Emotional impact and compassion fatigue in oncology nurses: Results of a multicentre study. European Journal of Oncology Nursing, v. 43, p. 101666, 1 dez. 2019.

ARMSTRONG, Karen. The great transformation: the beginning of our religious traditions. New York: Anchor Books, 2006. ISBN: 978-0-385-72124-0.

ASLAN, H.; ERCI, B.; PEKINCE, H. Relationship Between Compassion Fatigue in Nurses, and Work-Related Stress and the Meaning of Life. Journal of Religion and Health, 2 jan. 2021.

BATALHA, Edenise; MELLEIRO, Marta; BORGES, Elisabete. Satisfação e fadiga por compaixão em Enfermeiros da área hospitalar: resultados preliminares. International Congress of Occupational Health Nursing-ICOHN19: Proceedings. 2019. p. 87.

BORGES, E. M. DAS N. et al. Fadiga por compaixão em enfermeiros de urgência e emergência hospitalar de adultos. Revista Latino-Americana de Enfermagem, v. 27, 2019.

BHATTACHERJEE, Anol. Confiança individual em empresas online: desenvolvimento de escala e teste inicial. Journal of Management Information Systems , v. 19, n. 1, pág. 211 $241,2002$.

CAO, Xiaoyi; CHEN, Lin. The impact of resilience on turnover intention in dialysis nurses: The mediating effects of work engagement and compassion fatigue. Japan Journal of Nursing Science, p. e12414, 2021.

CAO, X. et al. Prevalence and predictors for compassion fatigue and compassion satisfaction in nursing students during clinical placement. Nurse Education in Practice, v. 51, p. 102999, 1 fev. 2021.

CÁCERES-RIVERA, D. I. Enfermería, pandemia y fatiga por compasión: una reflexión general sobre el 2020. Revista Ciencia y Cuidado, v. 18, n. 1, p. 116-123, 1 jan. 2021.

CROSS, L. A. Compassion Fatigue in Palliative Care Nursing. Journal of Hospice and Palliative Nursing, v. 21, n. 1, p. 21-28, fev. 2019.

DE OLIVEIRA, Vitória Alcântara; PASSOS, Marco Aurélio Ninomia. A IMPORTÂNCIA DOS PROFISSIONAIS ENFERMEIROS NA ASSISTÊNCIA AO COVID-19. Revista JRG de Estudos Acadêmicos, v. 3, n. 7, p. 367-378, 2020.

ESTEVES, G. G. L.; LEÃO, A. A. M.; ALVES, E. DE O. Fatigue and Stress as a predictors of Burnout in Health Care Professionals. Revista Psicologia Organizações e Trabalho, v. 19, n. 3, p. 695-702, set. 2019.

FORSYTH, Donelson R.; BURNETTE, Jeni. Processos de grupo. Advanced social 


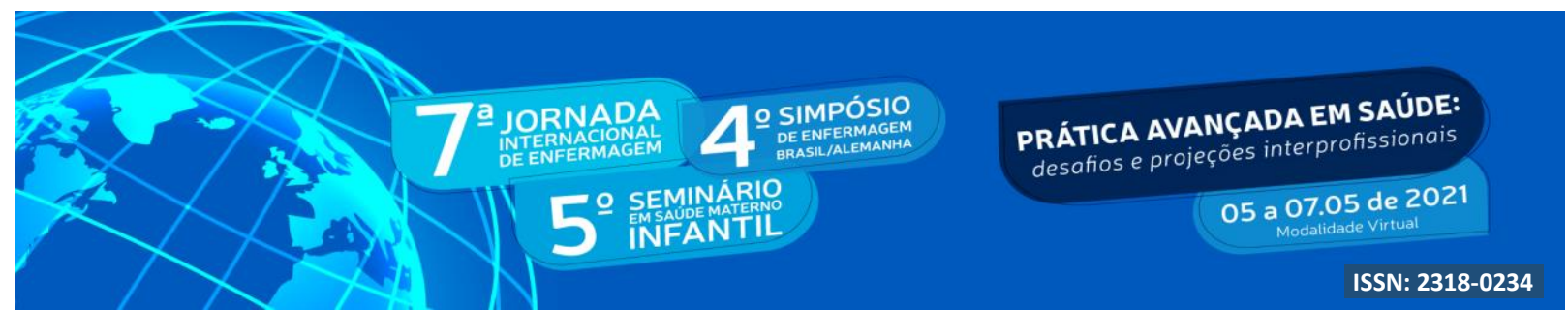

psychology: The state of the science, p. 495-534, 2010.

FUKUMORI, T. et al. Traumatic Events Among Cancer Patients That Lead to Compassion Fatigue in Nurses: A Qualitative Study. Journal of Pain and Symptom Management, v. 59, n. 2, p. 254-260, 1 fev. 2020.

HEGEL, J. et al. The Relationship Between Present-Centered Awareness and Attention, Burnout, and Compassion Fatigue in Oncology Health Professionals. Mindfulness, 5 Jan. 2021.

JIN, M. et al. Prevalence and factors of compassion fatigue among nurse in China. Medicine, v. 100, n. 3, 22 jan. 2021.

MARÇAL, Maria Teresa de Oliveira - A compaixão: uma dimensão ética do cuidar em enfermagem. Lisboa: Universidade Católica Portuguesa, Ciências de Enfermagem, 1994.

RINCÓN ÁLVAREZ, Daniel Andrés. Validade e confiabilidade da versão em espanhol do Instrumento de Competência Tecnológica como Cuidar em Enfermagem (TCCNI), Instrumento de Competência Tecnológica como Cuidado de Enfermagem. Faculdade de Enfermagem, 2013.

SCHULZE, B.; ANGERMEYER, M. C. Subjective experiences of stigma. A focus group study of schizophrenic patients, their relatives and mental health professionals. Social Science \& Medicine, v. 56, n. 2, p. 299-312, 1 Jan. 2003.

WENTZEL, D. L.; BRYSIEWICZ, P. A survey of compassion satisfaction, burnout and compassion fatigue in nurses practicing in three oncology departments in Durban, South Africa. International Journal of Africa Nursing Sciences, v. 8, p. 82-86, 1 Jan. 2018.

WHITTEMORE, Robin; KNAFL, Kathleen. The integrative review: updated methodology. Journal of advanced nursing, v. 52, n. 5, p. 546-553, 2005.

YU, H.; QIAO, A.; GUI, L. Predictors of compassion fatigue, burnout, and compassion satisfaction among emergency nurses: A cross-sectional survey. International Emergency Nursing, v. 55, p. 100961, 1 mar. 2021.

ZENKNER, K. V.; DENARDIN, E. F.; JESUS, A. A. de; STROM, B. R.; SILVA, E. S. da; CARLESSO, J. P. P. Mental health of health professionals: the illness of those who are dedicated to caring for the illness of others. Research, Society and Development, [S. l.], v. 9, n. 7, p. 47, 2020. 